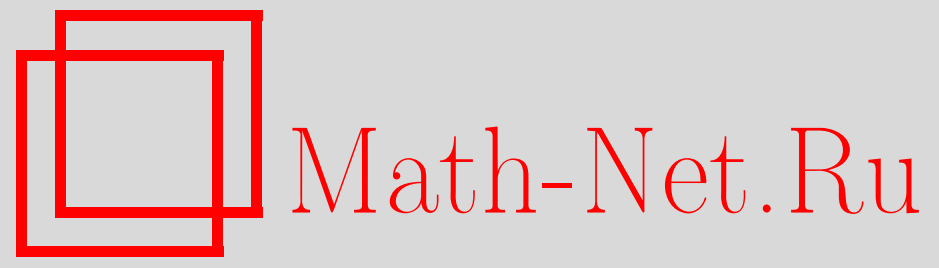

С. Глухова, Е. А. Палкин, Теория катастроф в статистическом анализе многомодальных распределений, Теория вероятн. и ее примен., 2004, том 49, выпуск 3, 485-502

DOI: https://doi.org/10.4213/tvp204

Использование Общероссийского математического портала MathNet.Ru подразумевает, что вы прочитали и согласны с пользовательским соглашением

http://www . mathnet.ru/rus/agreement

Параметры загрузки:

IP: 54.80 .73 .141

26 апреля 2023 г., $10: 32: 16$

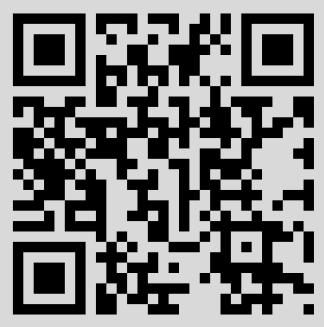




\title{
ТЕОРИЯ КАТАСТРОФ В СТАТИСТИЧЕСКОМ АНАЛИЗЕ МНОГОМОДАЛЬНЫХ РАСПРЕДЕЛЕНИЙ
}

\begin{abstract}
Рассматривается применение методов теории катастроф (классификации особенностей гладких отображений) для построения аналитических моделей объектов и процессов на основе статистических данных. Многомодальные одномерные статистические распределения сопоставлены с моделями катастроф коранга 1 - катастроф серии $A_{N}$. Предложены методы вычисления параметров катастроф типа $A_{N}$ (метод моментов и метод максимального правдоподобия) и их модификации применительно к случаям выраженных многомодальных и вырожденных квазиунимодальных распределений. Приведены результаты численных экспериментов по построению статистических моделей типа катастроф для случайных процессов.
\end{abstract}

Ключевые слова и фразы: многомодальные распределения, теория катастроф, параметрические семейства функций, метод моментов, метод максимального правдоподобия.

Введение. Применение методов теории катастроф в статистических исследованиях сложных объектов, описываемых многопараметрическими моделями, было предложено как одно из основных направлений уже в классических работах Р. Тома (см., например, [1]). Однако дальнейшее развитие прикладных методов теории катастроф более концентрировалось на качественных сторонах статистического анализа многомодальных распределений (см., например, двухтомник Р. Гилмора [2], работы В.И. Арнольда [3] и др., монографию Т. Постона и И. Стюарта [4] и цитируемую там литературу). Действительно, в многомодальньх распределениях каждый локальный максимум плотности распределения вероятности можно интерпретировать как одно из возможных значений случайной величины, реализующихся «устойчивым» образом в случайных выборках. Аналогичный смысл имеют критические точки семейств функций в теории катастроф. Поэтому если удается связать

* Главный военный клинический госпиталь им. акад. Н. Н. Бурденко, Госпитальная пл., 3, 105229 Москва, Россия; e-mail: nmc@a5.kiam.ru

** Московский физико-технический институт, Институтский пер., 9, 141700 г. Долгопрудный, Московская обл., Россия; e-mail: palkin@rosnow.ru 
наиболее вероятные значения случайной величины с критическими точками эталонных функций катастроф - универсальных деформаций особых ростков, то дальнейший анализ «поведения» случайной величины при изменении параметров модели можно выполнить с использованием стандартного математического аппарата теории катастроф [2]-[4]. В частности, можно исследовать такой важный эффект, как вырождение и трансформация мод многомодальных распределений. Кроме того, свойства типичности и устойчивости катастроф позволяют перенести эти их свойства и на многомодальные статистические распределения.

В рамках теории катастроф типичная задача статистической обработки данных сводится к следующему:

1) корректному выбору модели катастрофы (типу особенности);

2) оценке параметров модели в исследуемом диапазоне случайных величин;

3) оценке области применимости и достоверности построенного распределения.

Наиболее последовательно данная программа исследований отражена в работах [5]-[8]. Однако здесь многомодальное распределение априорно задается через полиномиальную функцию, отвечающую особенности типа $A_{N}$ (в работах зарубежных авторов такие катастрофы называют «каспоидными катастрофами»), в виде функции плотности условной вероятности

$$
f(x \mid \lambda)=\exp \left\{\theta-x^{N+1}+\sum_{j=1}^{N} \lambda_{j} x^{j}\right\},
$$

где $\lambda=\left(\lambda_{1}, \ldots, \lambda_{N}\right)$ - параметры распределения, $\theta$ - нормировочная константа, $N$ - кратность особенности, причем так как распределение (1) задано для $x \in \mathbf{R}^{1}$, то $N+1$ - четное. Поэтому открытым остается вопрос об адекватности модели (1) или иных моделей, предложенных в [5], и однозначности восстановления распределения по статистическим данным. В данной работе мы рассмотрим возможности и ограничения методов статистической обработки данных, имеющих выраженный многомодальный характер распределений, на основе моделей катастроф $A_{N}$, а также алгоритмы проверки гипотез о многомодальных распределениях типа (1).

1. Особенности $A_{N}$ как модели многомодальных одномерных статистических распределений. Рассмотрим параметрическое семейство гладких функций $f(x \mid y)$ (для простоты считаем, что $\left.f(x \mid y) \in C^{\infty}\right)$, задающих одномерную условную плотность вероятности $K$-модального вида, т.е. имеющих $K$ локальных максимумов: $x_{1}, \ldots, x_{K}$ и соответственно $K-1$ локальных минимумов $x_{1,2}, \ldots, x_{K-1, K}$ (рис. 1 ). Положение и значение локальных максимумов и минимумов зависит 
от параметров $y$. При каждом фиксированном значении $y$ для функции $f(x \mid y)$ выполняется условие нормировки:

$$
\int_{-\infty}^{+\infty} f(x \mid y) d x=1
$$

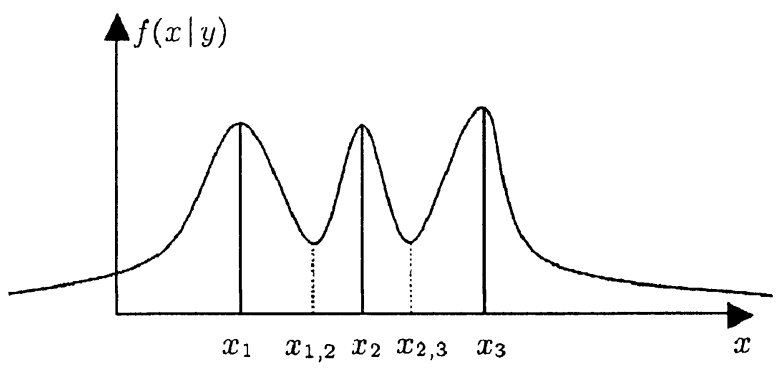

Рис. 1

Кроме того, $f(x \mid y)$ - строго неотрицательно определенная функция при любых $x$ и $y$ :

$$
f(x \mid y) \geqslant 0, \quad x \in \mathbf{R}^{1}, \quad y \in \mathbf{R}^{m} .
$$

Предположим, что сушествует значение параметра $y=y_{C}$, при котором все $2 K-1$ экстремальных точек функции $f(x \mid y)$ сливаются, образуя вырожденную критическую точку $x_{C}\left(y_{C}\right)$ кратности $2 K-1$. Данное условие исключает (хотя бы локально) влияние иных критических точек функции $f(x \mid y)$, имеющихся, возможно, для ее аналитического продолжения в $\mathbf{C}^{1}$. Вырожденная критическая точка имеется, очевидно, и у семейства функций

$$
\Phi(x, y)=\ln f(x \mid y) .
$$

Согласно теории катастроф, при сформулированных выше условиях в некоторой окрестности $U_{x} \otimes U_{y}$ внутренних переменных $x$ и внешних переменных $y$, содержащей точку $\left(x_{C}, y_{C}\right)$, существует гладкое невырожденное преобразование переменных $x$ :

$$
x=x(\xi, y), \quad \frac{\partial x}{\partial \xi} \neq 0
$$

такое, что

$$
\Phi(x, y)=F_{A_{2 K-1}^{-}}(\xi, \lambda(y))+\theta(y) .
$$

Здесь $F_{A_{2 K-1}^{-}}(\xi, \lambda(y))$ - универсальная деформация особого ростка параметрического семейства функций в вырожденной критической точке типа $A_{2 K-1}^{-}$,

$$
F_{A_{2 K-1}^{-}}(\xi, \lambda)=-\xi^{2 K}+\sum_{j=1}^{2 K-2} \lambda_{j} \xi^{j}, \quad \lambda \in \mathbf{R}^{2 K-2}, \quad K \geqslant 2,
$$


$\theta(y)$ - функция, не зависяшая от $\xi$. При этом вырожденная критическая точка $\left(x_{C}, y_{C}\right)$ соответствует значениям

$$
\lambda_{C}\left(y_{C}\right)=0, \quad \xi_{C}=0 .
$$

Знак «-» в (1.6) выбран из условия сходимости интеграла в (1.1). Предполагается также, что

$$
\frac{\partial x}{\partial \xi}>0
$$

Для функции $F_{A_{2 K-1}^{-}}(\xi, \lambda)(1.6)$, как и для функций $f(x \mid y), \Phi(x, y)$, существует область значений параметров $\lambda(y)$, где имеется $K$ локальных максимумов и $K-1$ локальных минимумов. Причем условия

$$
\begin{gathered}
\left.\frac{\partial f(x \mid y)}{\partial x}\right|_{x=x_{i}(y)}=0,\left.\quad \frac{\partial \Phi(x \mid y)}{\partial x}\right|_{x=x_{i}(y)}=0, \\
\left.\frac{\partial F_{A_{2 K-1}^{-}}(\xi, \lambda(y))}{\partial \xi}\right|_{\xi=\xi_{i}(\lambda(y))}=0,
\end{gathered}
$$

$i=1, \ldots, 2 K-1$, с учетом (1.4) эквивалентны.

Теорема 1. Если преобразование (1.4) K-модальной функиии плотности распределения вероятностей $f(x \mid y)$ может быть продолжено из области $U_{x} \otimes U_{y}$ на множество $\mathbf{R}_{x} \otimes U_{y}$, то функиия распределения вероятностей

$$
G\left(x^{*} \mid y\right)=\int_{-\infty}^{x^{*}} f(x \mid y) d x
$$

приводится $к$ виду

$$
\begin{aligned}
G\left(x^{*} \mid y\right) & =\int_{-\infty}^{\xi^{*}\left(x^{*}\right)} \exp \left\{F_{A_{2 K-1}^{-}}(\xi, \lambda(y))+\theta(y)\right\} \frac{\partial x}{\partial \xi} d \xi \\
& =l_{g, 0} G_{A_{2 K-1}}\left(\xi^{*}, \lambda\right)+\sum_{j=1}^{2 K-2} l_{g, j} \frac{\partial G_{A_{2 K-1}}}{\partial \lambda_{j}}\left(\xi^{*}, \lambda\right)+l_{e}
\end{aligned}
$$

2de

$$
G_{A_{2 K-1}}\left(\xi^{*}, \lambda\right)=\int_{-\infty}^{\xi^{*}} \exp \left\{F_{A_{2 K-1}^{-}}(\xi, \lambda)\right\} d \xi
$$

- каноническая функция $K$-модального распределения плотности вероятностей, а $l_{g, 0}, l_{g, 1}, \ldots, l_{g, 2 K-2}$ и $l_{e}$ - нормировочные, квазипостоянные (по отношению $\kappa G_{A_{2 K-1}}\left(\xi^{*}, \lambda\right)$ ) функции $\xi^{*} u \lambda$.

3 а мечания к те ореме 1.

1. Для практических вычислений фактически требуется существование и малость тех старших производных преобразования (1.4), которые используются для построения коэффициентов в разложении (1.11), а потому степень гладкости функции $f(x \mid y)$ и преобразования (1.4) может быть конечна. Порядок используемых в построении разложения (1.11) 
производных зависит от параметра $K$, т.е. от типа катастрофы. Их «малость» оценивается на основе формул (1.19), приведенных ниже.

2. В статистических задачах локальное преобразование (1.4) за пределы области $U_{x} \otimes U_{y}$ часто может быть продолжено любой подходящей функцией, если только данная область содержит все локальные экстремумы исследуемого распределения. Вклад случайных величин, выходящих за пределы этой ограниченной области, в статистических распределениях, как правило, пренебрежимо мал.

Доказательство теоремы 1 основано на следующей лемме.

Лемма 1. Функиии $G_{A_{2 K-1}}(\xi, \lambda)$ удовлетворяют каноническим дифференциальньм уравнениям:

$$
\begin{aligned}
2 K \frac{\partial^{2 K-1}}{\partial \lambda_{1}^{2 K-1}} G_{A_{2 K-1}}\left(\xi^{*}, \lambda\right)= & \sum_{j=2}^{2 K-2} j \lambda_{j} \frac{\partial^{j-1}}{\partial \lambda_{1}^{j-1}} G_{A_{2 K-1}}\left(\xi^{*}, \lambda\right) \\
& -\exp \left\{F_{A_{2 K-1}^{-}}\left(\xi^{*}, \lambda(y)\right)\right\},
\end{aligned}
$$

$2 \partial e$

$$
\begin{aligned}
\frac{\partial^{j}}{\partial \lambda_{1}^{j}} G_{A_{2 K-1}}\left(\xi^{*}, \lambda\right) & =\int_{-\infty}^{\xi^{*}\left(x^{*}\right)} \xi^{j} \exp \left\{F_{A_{2 K-1}^{-}}(\xi, \lambda(y))\right\} d \xi \\
& =G_{A_{2 K-1}, j}\left(\xi^{*}, \lambda\right)
\end{aligned}
$$

и справедливы соотношения $(j=1, \ldots, 2 K-2)$

$$
\frac{\partial^{j}}{\partial \lambda_{1}^{j}} G_{A_{2 K-1}}\left(\xi^{*}, \lambda\right)=\frac{\partial}{\partial \lambda_{j}} G_{A_{2 K-1}}\left(\xi^{*}, \lambda\right) .
$$

Доказательство леммы 1 следует из очевидного равенства

$$
\int_{-\infty}^{\xi^{*}\left(x^{*}\right)}\left[\frac{d}{d \xi} \exp \left\{F_{A_{2 K-1}^{-}}(\xi, \lambda)\right\}\right] d \xi=\exp \left\{F_{A_{2 K-1}^{-}}\left(\xi^{*}, \lambda\right)\right\}
$$

и определения функций $G_{A_{2 K-1, j}}\left(\xi^{*}, \lambda\right)$. Гладкость преобразования (1.4) во всей области $\mathbf{R}_{x} \otimes U_{y}$ позволяет разложить якобиан $\partial x / \partial \xi$ в подынтегральном выражении в (1.11) в степенной ряд по $\xi$. Тогда получим:

$$
G\left(x^{*} \mid y\right)=e^{\theta} \sum_{j=0}^{+\infty} b_{j} \int_{-\infty}^{\xi^{*}\left(x^{*}\right)} \xi^{j} \exp \left\{F_{A_{2 K-1}^{-}}(\xi, \lambda(y))\right\} d \xi
$$

где

$$
b_{j}(\lambda)=\left.\frac{1}{j !} \frac{\partial^{j+1} x}{\partial \xi^{j+1}}\right|_{\xi=0}
$$

В соответствии с уравнением (1.13) леммы 1, все члены в разложении (1.17) с $j \geqslant 2 K-1$ редуцируются к функциям типа (1.14) с $j=1, \ldots, 2 K-2$, что и доказывает теорему 1 . Этот алгоритм также позволяет построить явные выражения для функций $l_{g, 0}, l_{g, 1}, \ldots, l_{g, 2 K-2}$ и $l_{e}$. Проверка «квазипостоянности» этих функций является одновременно подтверждением корректности перехода (1.4), (1.5) к каноническому $K$-модальному распределению, т.е. корректности выбора модели катастрофы $A_{2 K-1}^{-}$. 
Аналогичные алгоритмы для построения асимптотических разложений, но для интегралов с осциллирующим экспоненциальным ядром, представляющих решения в методе канонического оператора Маслова и иных аналогичных методах, были рассмотрены в [9], [10]. В этих работах для однократных интегралов с особенностью типа $A_{N}$ у фазовой функции (аналогичной рассматриваемой здесь функции $\Phi(x, y)$ ) предложено использовать специальные функции волновых катастроф (CBK), связанные с краевыми катастрофами $B_{N}$. Такие CBK задаются интегралами с каноническим осциллирующим ядром и канонической областью интегрирования $[0,+\infty)$. В данной работе переход от канонических функций $K$-модальных распределений $G_{A_{2 K-1}}\left(\xi^{*}, \lambda\right)$ к эквивалентным функциям типа

$$
\widetilde{G}_{A_{2 K-1}}\left(\xi^{*}, \lambda\right)=\int_{0}^{+\infty} \exp \left\{-\xi^{N+1}+\sum_{j=1}^{N} \widetilde{\lambda}_{j}\left(\lambda, \xi^{*}\right) \xi^{j}\right\} d \xi
$$

аналогичным рассмотренным в [9], [10], не является оправданным ввиду специфики рассматриваемой задачи.

Используя алгоритм доказательства теоремы 1, запишем первые члены разложения нормировочных функций:

$$
\begin{aligned}
& l_{g, 0}=e^{\theta}\left\{\left.\frac{\partial x}{\partial \xi}\right|_{\xi=0}+\left.\frac{1}{(2 K) !} \frac{\partial^{2 K} x}{\partial \xi^{2 K}}\right|_{\xi=0} \lambda_{1}+\left.\frac{1}{2 K(2 K) !} \frac{\partial^{2 K+1} x}{\partial \xi^{2 K+1}}\right|_{\xi=0}+\cdots\right\}, \\
& l_{g, 1}=e^{\theta}\left\{\left.\frac{\partial^{2} x}{\partial \xi^{2}}\right|_{\xi=0}+\left.\frac{1}{(2 K) !} \frac{\partial^{2 K} x}{\partial \xi^{2 K}}\right|_{\xi=0} 2 \lambda_{2}\right. \\
& \left.+\left.\frac{1}{2 K(2 K) !} \frac{\partial^{2 K+1} x}{\partial \xi^{2 K+1}}\right|_{\xi=0} \lambda_{1}+\cdots\right\} \\
& l_{g, 2}=e^{\theta}\left\{\left.\frac{1}{2} \frac{\partial^{3} x}{\partial \xi^{3}}\right|_{\xi=0}+\left.\frac{1}{(2 K) !} \frac{\partial^{2 K} x}{\partial \xi^{2 K}}\right|_{\xi=0} 3 \lambda_{3}\right. \\
& \left.+\left.\frac{1}{2 K(2 K) !} \frac{\partial^{2 K+1} x}{\partial \xi^{2 K+1}}\right|_{\xi=0} 2 \lambda_{2}+\cdots\right\} \\
& l_{g, 2 K-3}=e^{\theta}\left\{\left.\frac{1}{(2 K-3) !} \frac{\partial^{2 K-2} x}{\partial \xi^{2 K-2}}\right|_{\xi=0}+\left.\frac{1}{(2 K) !} \frac{\partial^{2 K} x}{\partial \xi^{2 K}}\right|_{\xi=0}(2 K-2) \lambda_{2 K-2}\right. \\
& \left.+\left.\frac{1}{(2 K)} \frac{1}{(2 K) !} \frac{\partial^{2 K+1} x}{\partial \xi^{2 K+1}}\right|_{\xi=0}(2 K-3) \lambda_{2 K-3}+\cdots\right\} \\
& l_{g, 2 K-2}=e^{\theta}\left\{\left.\frac{1}{(2 K-2) !} \frac{\partial^{2 K-1} x}{\partial \xi^{2 K-1}}\right|_{\xi=0}\right. \\
& \left.+\left.\frac{1}{2 K} \frac{1}{(2 K) !} \frac{\partial^{2 K+1} x}{\partial \xi^{2 K+1}}\right|_{\xi=0}(2 K-2) \lambda_{2 K-2}+\cdots\right\}
\end{aligned}
$$




$$
\begin{aligned}
l_{e}= & -\exp \left\{\theta+F_{A_{2 K-1}^{-}}\left(\xi^{*}, \lambda(y)\right)\right\} \\
& \times\left\{\left.\frac{1}{(2 K) !} \frac{\partial^{2 K} x}{\partial \xi^{2 K}}\right|_{\xi=0}+\left.\frac{1}{2 K} \frac{1}{(2 K) !} \frac{\partial^{2 K+1} x}{\partial \xi^{2 K+1}}\right|_{\xi=0} \xi^{*}+\cdots\right\} .
\end{aligned}
$$

Из приведенных выражений видно, что квазипостоянность нормировочных функций, а также малость членов суммы в разложении (1.11) и функции $l_{e}$ по сравнению с $l_{g, 0}$ обеспечивается малостью старших производных функции $\partial x / \partial \xi$. В частности, формулы (1.19) дают возможность оценить и границы области $U_{x} \otimes U_{y}$, если в качестве центральной точки области взята особая точка $\left\{x_{C}, y_{C}\right\}$, соответствующая (в координатах $\xi, \lambda)$ точке $\xi=0, \lambda=0$.

Таким образом, исходная статистическая задача для случайной величины $x^{*}$ с функцией распределения (1.10) может быть сведена к «эталонной задаче» с функцией распределения (1.12), если:

(A) преобразование (1.4) продолжено из области $U_{x} \otimes U_{y}$ на множество $\mathbf{R}_{x} \otimes U_{y}$,

(B) нормировочные функции в разложении (1.11) не имеют особенностей при всех значениях $\xi^{*}\left(x^{*}\right), \lambda(y)$, «квазипостоянны» по отношению к функции $G_{A_{2 K-1}}\left(\xi^{*}, \lambda\right)$ и $l_{g, 0} \gg l_{g, j}, l_{e}, j=1, \ldots, 2 K-2$.

Проверка этих условий может быть выполнена только после построения преобразования (1.4). В тривиальном случае $x=\xi+\gamma(\gamma=$ const $)$, который фактически только и рассмотрен в работах [5]-[7], условия (A) и (В), очевидно, выполняются.

Будем считать, что в исследуемой задаче исходное распределение допускает преобразование к эталонному многомодальному распределению (1.12). Тогда множество наиболее вероятных значений $\xi_{n}$ связано с параметрами $y$ соотношением

$$
\begin{aligned}
\left.\frac{\partial F_{A_{2 K-1}^{-}}(\xi, \lambda(y))}{\partial \xi}\right|_{\xi=\xi_{n}} & =-2 K \xi^{2 K-1}+\sum_{j=1}^{2 K-2} j \lambda_{j} \xi_{n}^{j-1}=0 \\
\left.\frac{\partial^{2} F_{A_{2 K-1}^{-}}(\xi, \lambda(y))}{\partial \xi^{2}}\right|_{\xi=\xi_{n}} & =-2 K(2 K-1) \xi_{n}^{2 K-2}+\sum_{j=2}^{2 K-2} j(j-1) \lambda_{j} \xi_{n}^{j-2} \\
& =-\frac{1}{\widetilde{\varepsilon}_{k}(\lambda(y))}<0
\end{aligned}
$$

Уравнение (1.20) определяет $(2 K-2)$-мерное множество катастрофы $M$ в пространстве $\mathbf{R}_{\xi}^{1} \otimes \mathbf{R}_{\lambda}^{2 K-2}$ и, соответственно, $m$-мерное множество в пространстве $\mathbf{R}_{x}^{1} \otimes \mathbf{R}_{y}^{m}$. Причем условие (1.21) выделяет на этом множестве локальные максимумы функции плотности распределения вероятностей, отвечающие отдельным модам $\xi_{n}$ многомодального распределения. В качестве примера геометрия множества $M$ для $K=2$ (особенность $A_{3}$ ) представлена на рис. 2 . Темно-серая часть множества $M$ исключается условием (1.21). 


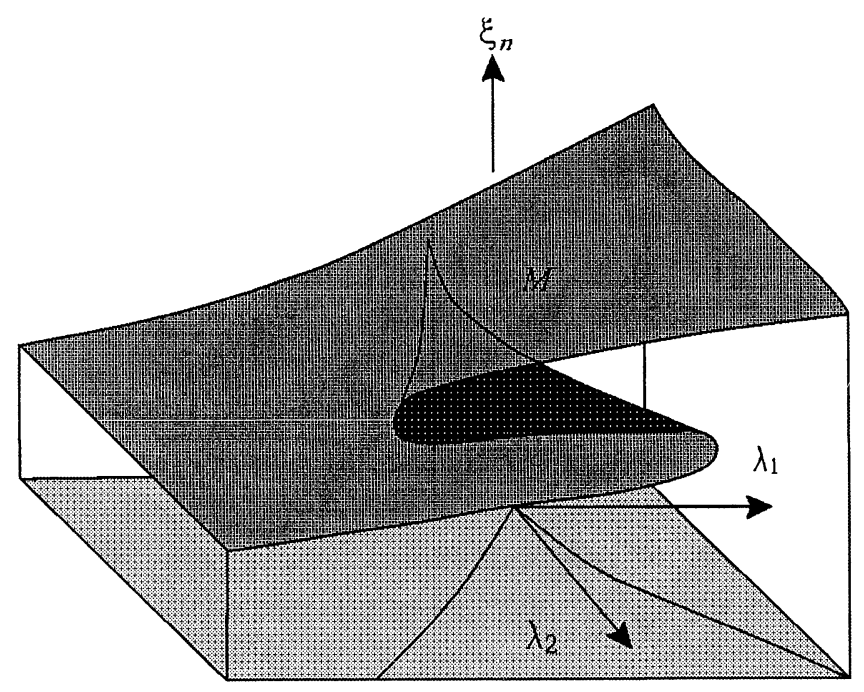

Рис. 2

В отличие от других задач, использующих аппарат теории катастроф, «равновесные» состояния статистической системы (множество $M$ ) не являются детерминированными. Реальная статистика в этом случае будет соответствовать точкам в пространстве $\xi_{n}, \lambda_{1}, \ldots, \lambda_{2 K-2}$, сосредоточенным в некоторой окрестности множества $M$.

В обычной одномерной статистической модели линейной регрессии существует одна зависимая переменная $x$ и произвольное число независимых переменных $y=\left(y_{1}, \ldots, y_{m}\right)$. Уравнение, описываюшее модель линейной регрессии, можно определить следующим образом:

$$
x(y)=b_{0}+\sum_{i=1}^{m} b_{i} y_{i}+\varepsilon,
$$

где $b_{i}-$ коэффициенты частной (линейной) зависимости $x$ от $y_{i}$ $(i=1, \ldots, m), \varepsilon-$ случайная величина, распределенная по нормальному закону с нулевым средним и неизвестной дисперсией. Регрессионная модель (1.22) имеет $m+2$ степени свободы. Множество наиболее вероятных значений величины $x$ (аналог множества $M(1.20)$ ) в соответствии с (1.22) определяет в этом случае гиперплоскость в пространстве $\mathbf{R}_{x}^{1} \otimes \mathbf{R}_{y}^{m}$.

По аналогии с линейной регрессионной моделью (1.22) определим статистическую модель катастрофы $A_{2 K-1}^{-}$линейной аппроксимацией зависимости внешних параметров $\lambda_{j}$ от $y$ :

$$
\lambda_{j}=\alpha_{0}^{j}+\sum_{i=1}^{m} \alpha_{i}^{j} y_{i}+\varepsilon_{j}, \quad j=1, \ldots, 2 K-2,
$$


и линейной аппроксимацией преобразования (1.4):

$$
\begin{aligned}
\xi & =\beta(x-\gamma) \\
\gamma & =\gamma_{0}+\sum_{i=1}^{m} \gamma_{i} y_{i}+\varepsilon
\end{aligned}
$$

где $\alpha_{0}^{j}, \alpha_{i}^{j}, \gamma$ и $\beta$ - не зависящие от $x$ величины $(\beta>0)$, а $\varepsilon_{j}, \varepsilon-$ случайные величины с характеристиками, аналогичными характеристикам величины $\varepsilon$ в $(1.22)$ для каждой компоненты $\lambda_{j}$ и параметра $\gamma$. Уравнение (1.20) определяет при этом нелинейную статистическую зависимость $x(y)$.

Для такой модели условия (A) и (В), очевидно, выполняются. Однако для априорно выбранной модели катастрофы выполнение равенства (1.5), как и область действия преобразований (1.23)-(1.25) требуют, как отмечалось выше, дополнительного исследования для оценки возможности их применения к статистическим данным конкретной задачи. Таким образом, выбор модели катастрофы $A_{2 K-1}^{-}$, точнее выбор числа $K$, на первом этапе представляет собой гипотезу о применимости данной модели. Ее обоснованием служит сходимость алгоритмов расчета аргументов функции $G_{A_{2 K-1}}\left(\xi^{*}, \lambda\right)$, а также коэффициентов в разложении (1.11). Важным критерием справедливости модели $A_{2 K-1}^{-}$ является немалость величины $\beta$, что гарантирует возможность применения линейной аппроксимации (1.24).

Статистическая задача для модели каспоидной катастрофы $A_{2 K-1}^{-}$ ставится, следовательно, таким образом: оценить коэффициенты в линейной зависимости $2 K-2$ внешних параметров $\lambda_{i}$ в $(1.23)$ и двух констант $\gamma$ и $\beta$ в (1.24), исходя из выборки, состоящей из статистических результатов «одновременного» наблюдения зависимой величины $x(y)$ и $m$ независимых переменных $\left(y_{1}, \ldots, y_{m}\right)$. Данная статистическая модель имеет $(2 K-1)(m+1)+1$ степеней свободы и при $K=1$, очевидно, переходит в линейную регрессионную модель (нормировочная константа $\beta$ вместе с величиной $\theta$ в (1.5) определяются из условия (1.1)). Отсутствие особенностей при решении этой задачи в представленных ниже алгоритмах (их внутренняя сходимость и устойчивость) будет указывать на корректность выбранной модели.

\section{2. Метод моментов для определения параметров много-} модальных распределений. Определение параметров универсальной деформации (1.6) и функции $\theta(y)$ в статистической модели (1.12), (1.23)-(1.25) можно провести различными способами. В данном пункте мы воспользуемся методом, предложенным в [5], распространив его на общий случай каспоидной катастрофы $A_{2 K-1}^{-}$. Система уравнений для определения величин $\alpha$ и $\gamma$ по заданной статистике внутренних $(x)$ и внешних $(y)$ переменных строится на основе следующей леммы [7]. 
Лемма 2. Пусть $f(x)$ - функиия плотности вероятности случайной величинь $x$, и пусть

$$
D(x)=-\frac{d}{d x} \ln f(x) .
$$

Тогда для любого полинома $p_{n}(x)$ степени $n$ математическое ожидание функиии $p_{n}(x) D(x)$ равно математическому ожиданию функчиu $\frac{d}{d x} p_{n}(x)$ :

$$
\mathbf{E}\left[p_{n}(x) D(x)\right]=\mathbf{E}\left[\frac{d p_{n}(x)}{d x}\right] .
$$

Доказательство очевидно.

Выбор функции (2.1), связанной с логарифмом плотности вероятности, для канонической функции $K$-модального распределения (1.12) сводит задачу непосредственно к расчету показателя экспоненты, т.е. к определению универсальной деформации. Необходимый (и достаточный) набор полиномов $p_{n}(x)$ определим, исходя из следующих свойств специальных функций $G_{A_{2 K-1}}\left(\xi^{*}, \lambda\right)$ (см. лемму 1 ).

Во-первых, отметим очевидное свойство канонических функций $K$-модальных распределений (1.12):

$$
\mathbf{E}\left[\xi^{n}\right](\lambda)=\lim _{\xi^{*} \rightarrow+\infty} \frac{G_{A_{2 K-1}, n}\left(\xi^{*}, \lambda\right)}{G_{A_{2 K-1}}\left(\xi^{*}, \lambda\right)}
$$

где $\mathbf{E}\left[\xi^{n}\right]$ - момент $n$-го порядка внутренней канонической переменной $\xi$.

Во-вторых, из уравнения (1.13) леммы 1 следует система рекуррентных соотношений для моментов старших порядков:

$$
\begin{aligned}
2 K \mathbf{E}\left[\xi^{2 K-1}\right](\lambda)= & \sum_{j=2}^{2 K-2} j \lambda_{j} \mathbf{E}\left[\xi^{j-1}\right](\lambda)+\lambda_{1}, \\
2 K \mathbf{E}\left[\xi^{2 K+n}\right](\lambda)= & \sum_{j=2}^{2 K-2} j \lambda_{j} \mathbf{E}\left[\xi^{j+n}\right](\lambda)+\lambda_{1} \mathbf{E}\left[\xi^{n+1}\right] \\
& +(n+1) \mathbf{E}\left[\xi^{n}\right], \quad n=0,1,2, \ldots .
\end{aligned}
$$

Таким образом, при фиксированных значениях $\lambda$ среди всех моментов величины $\xi$ имеется $2 K-2$ независимых.

Учитывая данные свойства моментов случайной величины $\xi$, в качестве полиномов $p_{n}(\xi)$ в (2.2) выберем такие, которые определяют независимые правые части в равенствах $(2.4),(2.5)$ и имеют минимальные порядки: мономы по $\xi$ порядков $n=0,1, \ldots, 2 K-1$ и мономы, содержащие линейную зависимость по $y_{i}$ (или $\lambda_{j}$ ) порядков $n=0,1, \ldots, 2 K-2$. То есть

$$
p_{n}: 1, \xi, \xi^{2}, \ldots, \xi^{2 K-1}, y_{i} \xi, y_{i} \xi^{2}, \ldots, y_{i} \xi^{2 K-1}, \quad i=1,2, \ldots, m .
$$


Используя набор мономов (2.6) в лемме 2 с учетом формулы (2.3), получим систему $(2 K-2)(m+1)+1$ нелинейных уравнений для нахождения параметров модели (1.23)-(1.25):

$$
\begin{aligned}
& \mathbf{E}[D(\xi)]=0, \\
& \mathbf{E}[\xi D(\xi)]=1, \\
& \mathbf{E}\left[\xi^{2} D(\xi)\right]=2 \frac{G_{A_{2 K-1,1}}(+\infty, \lambda)}{G_{A_{2 K-1}}(+\infty, \lambda)}, \\
& \mathbf{E}\left[\xi^{2 K-1} D(\xi)\right]=(2 K-1) \frac{G_{A_{2 K-1, K-2}}(+\infty, \lambda)}{G_{A_{2 K-1}}(+\infty, \lambda)}, \\
& \mathbf{E}\left[y_{i} D(\xi)\right]=0, \\
& \mathbf{E}\left[y_{i} \xi D(\xi)\right]=y_{i} \text {, } \\
& \mathbf{E}\left[y_{i} \xi^{2} D(\xi)\right]=2 y_{i} \frac{G_{A_{2 K-1,2}}(+\infty, \lambda)}{G_{A_{2 K-1}}(+\infty, \lambda)}, \\
& \mathbf{E}\left[y_{i} \xi^{2 K-2} D(\xi)\right]=(2 K-2) y_{i} \frac{G_{A_{2 K-1,2 K-3}}(+\infty, \lambda)}{G_{A_{2 K-1}}(+\infty, \lambda)},
\end{aligned}
$$

где

$$
D(\xi)=2 K \xi^{2 K-1}-\sum_{j=2}^{2 K-2} j \lambda_{j} \xi^{j-1}-\lambda_{1}
$$

Левые части уравнений системы (2.7) могут быть определены при известной статистике зависимости величины $x$ от внешних переменных $y_{i}$. Однако любая статистика будет содержать набор единичных, а не «многозначных» реализаций величины $x$ при заданных параметрах $y_{i}$. Поэтому в уравнениях системы следует провести вычисление левых частей при известном взаимном распределении зависимой случайной величины $x$ и независимых случайных параметров $y_{i}$. Характер таких статистических данных определяет два качественно различных вида системы (2.7).

1) Квазиунимодальное распределение статистических данньх. В этом случае функция $f(x \mid y)$ имеет вырожденное унимодальное распределение, существенно отличное от нормального или имеющее тенденцию к многомодальности, например, в силу какой-либо дополнительной информации об объекте исследования. Количество мод (индекс $K$ ) в этом случае может быть оценено априорно в рамках гипотезы о применимости модели катастрофы $A_{2 K-1}^{-}$на основе каких-либо косвенных данных. При выбранном $K$ можно построить эталонные $K$-модальные распределения методом моментов по данной статистике.

Будем считать, что случайные величины $y_{i}$ нормированы так, что

$$
\mathbf{E} y_{i}=0, \quad \mathbf{E} y_{i}^{2}=1, \quad i=1, \ldots, m \text {. }
$$


Проводя усреднение в системе (2.7) по значениям $y_{i}$, получим

$$
\begin{aligned}
& \mathbf{E}[D(\xi)]=0, \\
& \mathbf{E}[\xi D(\xi)]=1 \text {, } \\
& \mathbf{E}\left[\xi^{2} D(\xi)\right]=0 \text {, } \\
& \mathbf{E}\left[\xi^{n} D(\xi)\right] \\
& = \begin{cases}0 & \text { при } n \text { четном, } \\
n \Gamma\left(\frac{n}{2 K}\right)\left[\Gamma\left(\frac{1}{2 K}\right)\right]^{-1} & \text { при } n \text { нечетном, }\end{cases} \\
& \mathbf{E}\left[\xi^{2 K-1} D(\xi)\right]=(2 K-1) \Gamma\left(\frac{2 K-1}{2 K}\right)\left[\Gamma\left(\frac{1}{2 K}\right)\right]^{-1} ; \\
& \mathbf{E}\left[y_{i} D(\xi)\right]=0 \text {, } \\
& \mathbf{E}\left[y_{i} \xi D(\xi)\right]=0 \text {, } \\
& \mathbf{E}\left[y_{i} \xi^{2} D(\xi)\right]=0 \\
& \mathbf{E}\left[y_{i} \xi^{2 K-2} D(\xi)\right]=0 \text {. }
\end{aligned}
$$

При вычислении правых частей системы (2.10), кроме свойств (2.9), использовался тот факт, что вырожденная критическая точка катастрофы $A_{2 K-1}^{-}$для условий квазиунимодального распределения соответствует значению

$$
\mathbf{E}\left[\lambda_{j}\right]=0, \quad j=1, \ldots, 2 K-2 .
$$

В этом случае

$$
G_{A_{2 K-1, n}}(+\infty, \lambda)= \begin{cases}0 & \text { при } n \text { четном, } \\ \frac{1}{2 K} \Gamma\left(\frac{n+1}{2 K}\right) & \text { при } n \text { нечетном. }\end{cases}
$$

Условие (2.11), как правило, выполняется нестрого и фактически может быть проверено лишь только после решения системы (2.7) относительно $\lambda$. Поэтому решение системы (2.10) можно рассматривать либо как первую итерацию в решении системы (2.7), либо как оценку параметров модели (1.23)-(1.25). В работе [7] для оценки модели катастрофы «сборки» $(K=2)$ использовалась именно система типа (2.10).

2) K-модальное распределение статистических данных. Пусть теперь функция $f(x \mid y)$ имеет $K$ явно выраженных локальных максимумов, которые можно отождествить с $K$ нормально распределенными модами. При этом в качестве гипотезы используем предположение, что эти $K$ мод могут быть включены в единое $K$-модальное распределение, 
отвечающее канонической функции (1.12) катастрофы $A_{2 K-1}^{-}$. В этом случае

$$
G\left(x^{*} \mid y\right)=\sum_{k=1}^{K} G_{k}\left(x^{*} \mid y\right) \delta_{k}
$$

где $G_{k}\left(x^{*} \mid y\right)$ - функция распределения $k$-й моды с известными параметрами $x_{k}$ и $\varepsilon_{k}$ :

$$
G_{k}\left(x^{*} \mid y\right)=\frac{1}{\sqrt{2 \pi \varepsilon_{k}}} \int_{-\infty}^{x^{*}} \exp \left\{-\frac{\left(x-x_{k}\right)^{2}}{2 \varepsilon_{k}}\right\} d x
$$

и

$$
\sum_{k=1}^{K} \delta_{k}=1
$$

Тогда разложение (1.11) принимает вид:

$$
\sum_{k=1}^{K} G_{k}\left(x^{*} \mid y\right) \delta_{k}=l_{g, 0} G_{A_{2 K-1}}\left(\xi^{*}, \lambda\right)+\sum_{j=1}^{2 K-2} l_{g, j} \frac{\partial G_{A_{2 K-1}}}{\partial \lambda_{j}}\left(\xi^{*}, \lambda\right)+l_{e} .
$$

Равенство (2.16) предполагает, что функции $G_{A_{2 K-1}}$ и $G_{A_{2 K-1}, j}$ также могут быть представлены в виде суммы вкладов экстремальных точек (точнее, локальных максимумов) канонической плотности вероятности, число которых равно $K$. Проведя разложение этих функций на основе метода перевала, получаем формулы:

$$
\begin{aligned}
& G_{A_{2 K-1}}\left(\xi^{*}, \lambda\right) \cong \sum_{k=1}^{K} G_{A_{1}}^{k}\left(x^{*} \mid y\right) l_{0}^{k}+l_{e, 0} \\
& \frac{\partial G_{A_{2 K-1}}}{\partial \lambda_{j}}\left(\xi^{*}, \lambda\right) \cong \sum_{k=1}^{K} G_{A_{1}}^{k}\left(x^{*} \mid y\right) l_{j}^{k}+l_{e, j} .
\end{aligned}
$$

В оценках (2.17), (2.18) введены эталонные функции нормальных мод, отличающиеся от канонической функции $G_{A_{1}}\left(\xi^{*}, \lambda\right)$ наличием сдвигового параметра, что позволяет рассматривать их как функции единого аргумента $\xi^{*}$ :

$$
G_{A_{1}}^{k}\left(\xi^{*}, \lambda\right)=\int_{-\infty}^{\xi^{*}} \exp \left[-\frac{\left(\xi-\xi_{k}\right)^{2}}{2 \widetilde{\varepsilon}_{k}}\right] d \xi
$$

Сдвиговый параметр $\xi_{k}(\lambda)$ - «центр» моды, определяется как решения уравнение $(1.20), \widetilde{\varepsilon}_{k}$ определяется формулой (1.21). Коэффициенты $l_{0}^{k}, l_{j}^{k}, l_{e, 0}, l_{e, j}$ могут быть вычислены стандартным методом перевала для контура с концевой точкой. Сходимость такой вычислительной процедуры имеет особенности, аналогичные особенностям формулы (1.19), только в случае некорректного выбора модели катастрофы.

Подставляя $(2.17),(2.18)$ в $(2.16)$ и сравнивая левые и правые части по отдельным модам, приходим к системе равенств:

$$
G_{k}\left(x^{*} \mid y\right) \delta_{k}=G_{A_{1}}^{k}\left(\xi^{*}, \lambda\right) l^{k}, \quad k=1, \ldots, K
$$


Здесь $l^{k}-$ суммарный коэффициент $k$-й нормальной моды в переразложении (2.16) по формуле (2.19).

Применяя теперь метод моментов для каждой моды и используя в качестве функции $D(\xi)(2.1)$ в лемме $2 D_{k}(\xi)=\left(\xi-\xi_{k}\right) / \widetilde{\varepsilon}_{k}$, а в качестве функции $p_{n}$ мономы $1, \xi, y_{i}$, получим систему уравнений для определения параметров модели $(1.23)-(1.25)(k=1, \ldots, K)$ :

$$
\begin{aligned}
& \mathbf{E}^{k}\left[\frac{\xi-\xi_{k}}{\widetilde{\varepsilon}_{k}}\right]=0 \text { или } \quad \xi_{k}\left(x_{k}\right)=\mathbf{E}^{k}[\xi]=\xi_{k}(\lambda), \\
& \mathbf{E}^{k}\left[\xi \frac{\xi-\xi_{k}}{\widetilde{\varepsilon}_{k}}\right]=1 \quad \text { или } \quad \widetilde{\varepsilon}_{k}\left(x_{k}\right)=\mathbf{E}^{k}\left[\left(\xi-\xi_{k}\right)^{2}\right]=\widetilde{\varepsilon}_{k}(\lambda) .
\end{aligned}
$$

При $\beta>0$ можно провести нормировку и считать, что $\beta=1$ в (1.24), тогда $\xi_{k}=x_{k}-\mathbf{E}^{k}[\gamma], \widetilde{\varepsilon}_{k}=\varepsilon_{k}$. Система $2 K$ уравнений $(2.21)$ и (2.22) позволяет определить средние значения параметров $\lambda, \gamma$ в модели (1.23)-(1.25). Их зависимость от $y_{i}$ находится из следующих уравнений:

$$
\begin{aligned}
\mathbf{E}^{k}\left[y_{i} \frac{\xi-\xi_{k}}{\widetilde{\varepsilon}_{k}}\right] & =0 \quad \text { или } \\
\mathbf{E}^{k}\left[y_{i}\left(\xi-\xi_{k}(\lambda(y))\right)\right] & =0, \quad k=1, \ldots, K, \quad i=1, \ldots, m .
\end{aligned}
$$

Однако число уравнений (2.23) $K m$ меньше числа искомых параметров линейной зависимости $(2 K-1) m$. «Неполнота» системы $(2.21)-(2.23)$ обусловлена тем, что среди $2 K-1$ критических точек канонической функции распределения имеется $K-1$ точек локальных минимумов, которые в рассматриваемой $K$-модальной ситуации оказываются информационно незначимыми.

Для обеспечения полноты системы воспользуемся статистикой параметра $y_{i}$. Взяв в качестве монома $p_{n}=y_{i} \xi$, из формулы $(2.2)$ получаем

$$
\mathbf{E}^{k}\left[y_{i} \xi \frac{\xi-\xi_{k}}{\widetilde{\varepsilon}_{k}}\right]=\mathbf{E}^{k}\left[y_{i}\right], \quad k=1, \ldots, K,
$$

где $\mathbf{E}^{k}\left[y_{i}\right]$ - среднее значение параметра $y_{i}$ в $k$-й моде. В силу $(2.13)$ и $(2.15)$

и, следовательно,

$$
\mathbf{E}\left[y_{i}\right]=\sum_{k=1}^{K} \mathbf{E}^{k}\left[y_{i}\right] \delta_{k}
$$

$$
\mathbf{E}^{k}\left[y_{i} \xi \frac{\xi-\xi_{k}}{\widetilde{\varepsilon}_{k}}\right] \delta_{k^{\prime}}=\mathbf{E}^{k^{\prime}}\left[y_{i} \xi \frac{\xi-\xi_{k^{\prime}}}{\widetilde{\varepsilon}_{k^{\prime}}}\right] \delta_{k}, \quad k, k^{\prime}=1, \ldots, K, \quad k \neq k^{\prime} .
$$

Соотношения (2.26) определяют $(K-1) m$ независимых уравнений, которые дополняют систему (2.21)-(2.23) и позволяют определить все параметры модели (1.23)-(1.25).

В качестве примера построим систему метода моментов для бимодального распределения $(K=2)$ и соответствующей статистической модели катастрофы $A_{3}^{-}$в квазиунимодальном приближении. 
Для простоты будем считать, что $\gamma_{i}=0$ в (1.25). Тогда

$$
\begin{aligned}
f(x \mid y)=\exp \{ & \theta-\beta^{4}\left(x-\gamma_{0}\right)^{4}+\beta^{2}\left(\alpha_{0}^{2}+\sum_{i=1}^{m} \alpha_{i}^{2} y_{i}\right)\left(x-\gamma_{0}\right)^{2} \\
& \left.+\beta\left(\alpha_{0}^{1}+\sum_{i=1}^{m} \alpha_{i}^{1} y_{i}\right)\left(x-\gamma_{0}\right)\right\} .
\end{aligned}
$$

Соответственно,

$$
\begin{aligned}
D\left(x, a^{1}, a^{2}, y\right)= & {\left[4 \beta^{4}\left(x-\gamma_{0}\right)^{3}-2 \beta^{2}\left(\alpha_{0}^{2}+\sum_{i=1}^{m} \alpha_{i}^{2} y_{i}\right)\left(x-\gamma_{0}\right)\right.} \\
& \left.-\beta\left(\alpha_{0}^{1}+\sum_{i=1}^{m} \alpha_{i}^{1} y_{i}\right)\right] .
\end{aligned}
$$

Раскроем скобки, сгруппируем члены при мономах $x^{3}, x^{2}, x, x y_{i}, y_{i}$ и введем следующие обозначения:

$$
\begin{aligned}
b_{1} & =\left[2 \beta^{2} \alpha_{0}^{2} \gamma_{0}-\beta \alpha_{0}^{1}-4 \beta^{4} \gamma_{0}^{3}\right], & b_{2} & =\left[12 \beta^{4} \gamma_{0}^{2}-2 \beta^{2} \alpha_{0}^{2}\right], \\
b_{1+2 i} & =2 \beta^{2} \alpha_{i}^{2} \gamma_{0}-\beta \alpha_{i}^{1}, & b_{2+2 i} & =-2 \beta^{2} \alpha_{i}^{2}, \\
b_{3+2 m} & =-12 \beta^{4} \gamma_{0}, & b_{4+2 m} & =4 \beta^{4} .
\end{aligned}
$$

$\mathrm{B}$ этих обозначениях $D\left(x, a^{1}, a^{2}, y\right)=b_{1}+b_{2} x+\sum_{i=1}^{m} b_{1+2 i} y_{i}+$ $\sum_{i=1}^{m} b_{2+2 i} y_{i} x+b_{3+2 m} x^{2}+b_{4+2 m} x^{3}$. В соответствии с $(2.6)$ в качестве $p_{n}$ выберем мономы:

$$
\begin{gathered}
p_{1}=1, \quad p_{2}=\xi, \quad p_{1+2 i}=y_{i}, \quad p_{2+2 i}=y_{i} \xi, \quad i=1, \ldots, m, \\
p_{3+2 m}=\xi^{2}, \quad p_{4+2 m}=\xi^{3} .
\end{gathered}
$$

Применяя лемму 2 и набор выбранных функций, получаем систему уравнений (2.7), которую можно представить в виде:

$$
L b=a,
$$

где элементы $a$, за исключением трех:

$$
a_{2}=\frac{1}{\beta}, \quad a_{3+2 m}=\frac{2 \gamma_{0}}{\beta}, \quad a_{4+2 m}=\frac{3}{\beta}\left[\frac{\Gamma\left(\frac{3}{4}\right)}{\beta^{2} \Gamma\left(\frac{1}{4}\right)}+\gamma_{0}^{2}\right],
$$

равны нулю. Считается, что все случайные величины приведены к стандартному виду (нулевому среднему и стандартному отклонению, равному единице). Этот шаг значительно упрошает вычисления и улучшает их численную точность.

Матрица $L$, имеющая размерность $(2 m+4) \times(2 m+4)$, состоит из элементов вида

$$
L_{i j}=\mathbf{E}\left[\omega_{i} \omega_{j}\right]_{i, j=1, \ldots, 2 m+4}
$$

где $\omega_{1}=1, \omega_{2}=x, \omega_{1+2 i}=y_{i}, \omega_{2+2 i}=y_{i} x, i=1, \ldots, m, \omega_{3+2 m}=x^{2}$, $\omega_{4+2 m}=x^{3}$. Правая часть в формуле (2.31) есть среднее значение соответствующих величин, определенное в квазиунимодальном распределении данных наблюдений. 
Из (2.30) вектор $b$ определяется как $b=L^{-1} a$. Далее, из (2.29) находим параметры модели (1.23)-(1.25):

$$
\begin{aligned}
\beta & =\left(\frac{1}{4} b_{4+2 m}\right)^{1 / 4}, \quad \gamma_{0}=-\frac{b_{3+2 m}}{3 b_{4+2 m}}, \\
\alpha_{0}^{1} & =\left(\frac{1}{4} b_{4+2 m}\right)^{-1 / 4}\left[\frac{b_{3+2 m}^{2}}{3 b_{4+2 m}}\left(b_{2}-\frac{2}{9} \frac{b_{3+2 m}^{2}}{b_{4+2 m}}\right)-b_{1}\right], \\
\alpha_{0}^{2} & =\left(b_{4+2 m}\right)^{-1 / 2}\left[\frac{b_{3+2 m}^{2}}{b_{4+2 m}}-b_{2}\right], \\
\alpha_{i}^{1} & =\left(\frac{1}{4} b_{4+2 m}\right)^{-1 / 4}\left[\frac{b_{3+2 m}^{2}}{3 b_{4+2 m}} b_{2+2 i}-b_{1+2 i}\right], \quad \alpha_{i}^{2}=-\frac{b_{2}}{\left(b_{4+2 m}\right)^{1 / 2}} .
\end{aligned}
$$

Решение системы (2.30) существует только при $\beta \neq 0$, что доказывает справедливость модели катастрофы $A_{3}^{-}$. В свою очередь, данное условие соответствует $b_{4+2 m} \neq 0$, что обеспечивает корректность формул для искомых коэффициентов $\gamma_{0}, \alpha_{i}^{1}, \alpha_{i}^{2}(i=0,1, \ldots, m)$.

3. Метод максимального правдоподобия. Метод максимального правдоподобия является наиболее распространенным методом оценки достоверности моделей статистических распределений или значений параметров параметрических моделей. Рассмотрим этот метод применительно к задаче определения параметров моделей катастроф типа $A_{N}(1.11),(1.23)-(1.25)$.

Пусть $x^{j}, y_{i}^{j}, i=1, \ldots, m,-$ выборка объема $J(j=1, \ldots, J)$ из генеральной совокупности независимых переменных - компонент вектора $y$ и зависимой переменной $x$ с теоретической функцией распределения вида (1.11). Эта выборка статистических данных представляет набор точек, сосредоточенных в окрестности частей множества катастрофы $M$, допускаемых условием (1.21). Так как такие точки отвечают максимумам функции плотности распределения вероятностей $f(x \mid y)$, то в в качестве функции правдоподобия можно взять параметрическое семейство функций вида:

$$
\begin{aligned}
P\left(x^{j}\left(y^{j}\right), y^{j}\right) & =\ln \prod_{j=1}^{J} \exp \left[F_{A_{2 K-1}^{-}}\left(\xi\left(x^{j}\right), \lambda\left(y^{j}\right)\right)+\theta\left(y^{j}\right)\right] \\
& =\sum_{j=1}^{J}\left[F_{A_{2 K-1}^{-}}\left(\xi\left(x^{j}\right), \lambda\left(y^{j}\right)\right)+\theta\left(y^{j}\right)\right] .
\end{aligned}
$$

Параметры $\alpha_{i}^{k}, \gamma_{i}(i=0,1, \ldots, m, k=1, \ldots, 2 K-2), \beta$ находятся из условия максимума функции правдоподобия и являются решениями системы нелинейных уравнений:

$$
\frac{\partial P}{\partial \alpha_{i}^{k}}=0, \quad \frac{\partial P}{\partial \gamma_{i}}=0, \quad \frac{\partial P}{\partial \beta}=0 .
$$


Данная система нелинейных уравнений решается с использованием численных методов. Сходимость численного решения будет критерием справедливости модели катастрофы $A_{2 K-1}^{-}$.

В случае катастрофы сборки $A_{3}^{-}$функция правдоподобия имеет вид:

$$
\begin{aligned}
& P\left(x^{j}\left(y^{j}\right), y^{j}\right)=\sum_{j=1}^{J}\left[\theta_{j}+\left(\alpha_{0}^{1}+\sum_{i=1}^{m} \alpha_{i}^{1} y_{i}^{j}\right)\left(x^{j}-\gamma_{0}-\sum_{i=1}^{m} \gamma_{i} y_{i}^{j}\right)\right. \\
& \left.+\left(\alpha_{0}^{2}+\sum_{i=1}^{m} \alpha_{i}^{1} y_{i}^{j}\right)\left(x^{j}-\gamma_{0}-\sum_{i=1}^{m} \gamma_{i} y_{i}^{j}\right)^{2}-\beta\left(x^{j}-\gamma_{0}-\sum_{i=1}^{m} \gamma_{i} y_{i}^{j}\right)\right]^{4} .
\end{aligned}
$$

Соответственно, систему (3.2) представляют следующие уравнения:

$$
\begin{aligned}
\frac{\partial P}{\partial \alpha_{0}^{1}}= & \sum_{j=1}^{J}\left(\frac{\partial \theta_{j}}{\partial \alpha_{0}^{1}}+\left(x^{j}-\gamma_{0}-\sum_{i=1}^{m} \gamma_{i} y_{i}^{j}\right)\right)=0, \\
\frac{\partial P}{\partial \alpha_{i}^{1}}= & \sum_{j=1}^{J}\left(\frac{\partial \theta_{j}}{\partial \alpha_{i}^{1}}+y_{i}^{j}\left(x^{j}-\gamma_{0}-\sum_{i=1}^{m} \gamma_{i} y_{i}^{j}\right)\right)=0, \\
\frac{\partial P}{\partial \alpha_{0}^{2}}= & \sum_{j=1}^{J}\left(\frac{\partial \theta_{j}}{\partial \alpha_{0}^{2}}+\left(x^{j}-\gamma_{0}-\sum_{i=1}^{m} \gamma_{i} y_{i}^{j}\right)^{2}\right)=0, \\
\frac{\partial P}{\partial \alpha_{i}^{2}}= & \sum_{j=1}^{J}\left(\frac{\partial \theta_{j}}{\partial \alpha_{i}^{2}}+y_{i}^{j}\left(x^{j}-\gamma_{0}-\sum_{i=1}^{m} \gamma_{i} y_{i}^{j}\right)^{2}\right)=0, \\
\frac{\partial P}{\partial \gamma_{0}}= & \sum_{j=1}^{J}\left(\frac{\partial \theta_{j}}{\partial \gamma_{0}}-\left(\alpha_{0}^{1}+\sum_{i=1}^{m} \alpha_{i}^{1} y_{i}^{j}\right)-2\left(\alpha_{0}^{2}+\sum_{i=1}^{m} \alpha_{i}^{2} y_{i}^{j}\right)\right. \\
& \left.\times\left(x^{j}-\gamma_{0}-\sum_{i=1}^{m} \gamma_{i} y_{i}^{j}\right)+4 \beta\left(x^{j}-\gamma_{0}-\sum_{i=1}^{m} \gamma_{i} y_{i}^{j}\right)^{3}\right)=0, \\
\frac{\partial P}{\partial \gamma_{i}}= & \sum_{j=1}^{J}\left(\frac{\partial \theta_{j}}{\partial \gamma_{i}}-y_{i}^{j}\left(\alpha_{0}^{1}+\sum_{i=1}^{m} \alpha_{0}^{1} y_{i}^{j}\right)-y_{i}^{j}\left(\alpha_{0}^{2}+\sum_{i=1}^{m} \alpha_{i} y_{i}^{j}\right)\right. \\
& \left.\quad \times\left(x^{j}-\gamma_{0}-\sum_{i=1}^{m} \gamma_{i} y_{i}^{j}\right)+4 \beta y_{i}^{j}\left(x^{j}-\gamma_{0}-\sum_{i=1}^{m} \gamma_{i} y_{i}^{j}\right)^{3}\right)=0, \\
\frac{\partial P}{\partial \beta}= & \sum_{j=1}^{J}\left(\frac{\partial \theta_{j}}{\partial \beta}-\left(x^{j}-\gamma_{0}-\sum_{i=1}^{m} \gamma_{i} y_{i}^{j}\right)^{4}\right)=0 .
\end{aligned}
$$

Системы уравнений метода максимального правдоподобия имеют, как правило, несколько решений и потому могут использоваться лишь для уточнения параметров модели. Первичная оценка значений параметров может быть найдена, например, методом моментов.

Заключение. Рассмотренные в работе методы оценки параметров моделей многомодальных статистических распределений, использующих канонические формы теории катастроф, являются важными в прикладных аспектах, поскольку позволяют применить при анализе состояний 
исследуемого объекта весь современный аппарат этой теории. Данный подход позволяет прогнозировать поведение объекта не только в области значений имеющихся статистических данных, но и за пределами этой области. В этой (нелинейной) экстраполяции важным вопросом является достоверность «продолжения» модели катастрофы за пределы области значений статистических данных. Теоретическое рассмотрение этой задачи является предметом отдельного исследования. Некоторые оценочные заключения о размерах области применимости рассматриваемых здесь моделей катастроф $A_{2 K-1}^{-}$можно получить, если применить метод кластеризации данных, а затем использовать прямые вычисления параметров единой модели для различных групп данных и сопоставить полученные результаты экстраполяции параметров из групп в группу.

Другим альтернативным методом проверки качества модели может служить восстановление параметров при численном моделировании статистических распределений с априорно заданной функцией плотности распределения вероятностей. Этот прием был нами реализован для сравнения эффективности метода моментов и метода максимального правдоподобия для модели катастрофы $A_{3}^{-}$. Результаты расчетов показали пригодность обоих методов для построения моделей типа катастроф. При этом для квазиунимодальных распределений метод максимального правдоподобия оказался более точным, что связано со значительными погрешностями приближенных уравнений (2.9), (2.10) метода моментов.

\section{СПИСОК ЛИТЕРАТУРЫ}

1. Thom R. Structural Stability and Morphogenesis: An Outline of a General Theory of Models. - Reading: Benjamin, 1975, 348 p.

2. Гилмор Р. Прикладная теория катастроф. Т. 1. М.: Мир, 1984, 350 с.

3. Арнольд В. И. Теория катастроф. М.: Наука, 1990, 128 с.

4. Постон Т., Стюарт И. Теория катастроф и ее приложения. М.: Мир, 1980, 607 c.

5. Cobb L., Koppstein P., Chen N.H. Estimation and moment recursion relations for multimodal distributions of the exponential family. - J. Amer. Statist. Assoc., 1983, v. 78 , № 381, p. 124-130.

6. Cobb L., Zacks S. Applications of catastrophe theory for statistical modeling in the biosciences. - J. Amer. Statist. Assoc., 1985, v. 80, № 392, p. 793-802.

7. Cobb L. Parameter estimation for the cusp catastrophe model. - Behavioral Sci., 1981, v. 26 , p. $75-78$.

8. Oliva T.A., Desarbo W.S., Day D.L., Jedidi K. GEMCAT: a general multivariate methodology for estimating catastrophe models. - Behaviorial Sci., 1987, v. 32, № 2, p. $121-137$.

9. Ипатов Е. Б., Крюковский А. С., Лукин Д. С., Палкин Е. А. Краевые катастрофы и асимпітотики. - Докл. АН СССР, 1986, т. 291, № 4, с. 823-827.

10. Крюковский A.C., Лукин Д. С., Палкин Е. А. Краевые и угловые катастрофы в задачах дифракции и распространения волн. Казань: Казанский авиационный ин-т, 1988, 199 с. 